\title{
Petechiae: Reproducible Pattern of Distribution and Increased Appearance After Bed Rest
}

\author{
Bergita Ganse, Ulrich Limper, Judith Bühlmeier, \\ AND JÖRN RITTWEGER
}

\begin{abstract}
Ganse B, Limper U, BüHLMeIER J, RitTweger J. Petechiae: reproducible pattern of distribution and increased appearance after bed rest. Aviat Space Environ Med 2013; 84:864-6.

Background: Exposure to acceleration can cause petechial hemorrhages, called G measles. Petechiae usually start to develop between 5 and $9 \mathrm{G}$ with a high interindividual variance. Centrifuge training delays the onset to higher $G$ levels. One might expect onset at lower $\mathrm{G}$ levels after bed rest; however, there is no evidence in the literature. A case of petechiae formation after bed rest is presented here. Case report: Orthostatic tolerance was tested using a tilt table and lower body negative pressure before and after bed rest in both campaigns of a $2 \times 21-d$ bed rest study with $6^{\circ}$ head-down tilt. A 42 -yr-old male Caucasian without any history of thrombosis, venous disease, hemorrhage, or petechiae, and with a negative thrombophilia screening, took part in the bed rest study as 1 out of 10 subjects. He was the only one to develop petechiae during the orthostatic tests after, but not before, bed rest in both campaigns. Petechiae were distributed throughout the lower legs and most pronounced at the shin in a stocking-like fashion, surprisingly reoccurring in an identical pattern of distribution. Petechiae appeared slowly over minutes during hyperemia. Discussion: This case indicates that prolonged bed rest decreases the threshold for petechiae formation. A reproducible distribution pattern suggests that factors predisposing to petechiae formation keep their local distribution over time (possibly due to local vessel structures). Mechanisms of adaptation and interindividual variance are unclear. Findings are of clinical relevance as such cases might occur after prolonged bed rest in patients without need of expensive testing.

Keywords: G measles, petechial hemorrhages, acceleration, tilt table, LBNP.
\end{abstract}

WELL-KNOWN CAUSES for petechial hemorrhage include infectious diseases, vasculitis, thrombocytopenia, tumors, and physical pressure, e.g., in strangulation or reanimation. It is less widely appreciated, however, that exposure to acceleration often induces 'G measles' in astronauts $(5,6)$, fighter pilots, bungeejumpers, in centrifugation (8), and also in daily life, including rollercoaster rides and car accidents. Petechiasis usually starts to develop between $5 \mathrm{G}$ and $9 \mathrm{G}$ in those parts of the human body where pressure load is highest (7). Astronauts regularly develop petechiae in the back integument during the course of descent in the Soyuz capsule after long-term spaceflight $(5,6)$. Reasons for the substantial interindividual variation in the occurrence are not understood. Increased vascular fragility and permeability are discussed, resulting in either capillary rupture or exudation. Effects of hypergravity on hemostasis are known, but it is unclear how far they influence petechiae formation (3). An acclimatized resistance can be induced by centrifuge training, which means that onset is delayed to higher $G$ levels $(4,7)$. One might expect the reverse, namely increased likelihood of petechiasis, to happen after prolonged bed rest. A text book from 1974 states this as given fact; however, unfortunately without giving material evidence (1). Descriptions of this phenomenon can otherwise not be found in the literature. During the past 10 years petechiasis has not been observed in the more than 100 young healthy subjects who underwent experimental bed rest for periods between 5 and $90 \mathrm{~d}$ in the German Aerospace Center. Now, however, a case of petechiae formation has occurred during tilt table testing, which is presented here. This case reveals several new aspects that might lead to a better understanding of petechial hemorrhages in general.

\section{CASE REPORT}

The study protocol was approved in advance by the ethics committee of North Rhine Medical Association (No. 2010426). Each subject provided written informed consent before participating. The bed rest study was performed in $6^{\circ}$ head-down tilt with two campaigns of $21 \mathrm{~d}$ bed rest 4 mo apart. A randomized cross-over design was used to test a nutritional intervention with whey protein and alkaline mineral salts.

There were 10 male subjects who participated in the study with an average age of $31.6 \pm 6.2 \mathrm{yr}$ at the beginning of the first campaign. Subjects did not receive medications altering blood coagulation such as heparin or warfarin. An orthostatic test was performed before and after bed rest in each campaign using a tilt table and lower body negative pressure (LBNP). Lower body negative pressure is a standard means in gravitational physiology to augment gravity's pull on the vascular system (2). After $30 \mathrm{~min}$ of $6^{\circ}$ head-down baseline data collection,

From the German Aerospace Center, Institute of Aerospace Medicine, Division of Space Physiology, Cologne, Germany; the University Witten/ Herdecke, Department of Anaesthesiology and Operative Intensive Care Medicine, Medical Center Cologne-Merheim, Cologne, Germany; and the University of Bonn, Department of Nutrition and Food Science, Bonn, Germany.

This manuscript was received for review in October 2012. It was accepted for publication in January 2013.

Address correspondence and reprint requests to: Dr. med. Bergita Ganse, Division Space Physiology, German Aerospace Center, Institute of Aerospace Medicine, Porz-Wahnheide, Linder Höhe, D-51147 Cologne, Germany; bergita.ganse@dlr.de.

Reprint \& Copyright (C) by the Aerospace Medical Association, Alexandria, VA.

DOI: $10.3357 /$ ASEM.3567.2013 
the subject was tilted to $80^{\circ}$ head-up for $15 \mathrm{~min}$, followed by graded LBNP increasing by $-10 \mathrm{mmHg}$ of pressure every 3 min until signs of presyncope occurred. The test was stopped as soon as any of the following criteria were fulfilled: systolic blood pressure $<70 \mathrm{mmHg}$; decrease in systolic blood pressure of $>25 \mathrm{mmHg}$; sudden drop in heart rate greater than $15 \mathrm{bpm}$; premature ventricular contractions (changes from baseline); sudden and rapidly progressive onset of or change in sweating, pallor, yawning, anxiety, stomach awareness, nausea, dizziness, tingling, tunnel vision, or other sensations unusual to the subject; undue delay or lack of subject response to questions; fainting; subject asking to terminate the test; or decision of the medical monitor.

The test subject was a 42-yr-old male Caucasian without any history of thrombosis, venous disease, hemorrhage, or petechiae, and with a negative thrombophilia screening. He did a reasonable amount of exercise in his normal life, including weight training 1-3 times per week and long walks with his dogs on a daily basis, and could therefore be considered an athletic type. Furthermore, the subject worked as a commercial clerk in a chemical company with regular working hours and no shift work. His bodyweight was $74.39 \mathrm{~kg}$ at the start of the first campaign (average bodyweight of all subjects: $76.11 \pm 5.46 \mathrm{~kg}$ ). During subject selection, laboratory examinations were within normal limits, including PT, INR, PPT, Factors I and II, Factor V Leyden, AT III, Lupus-PTT, thrombocyte count, and platelet function tests.

The subject developed petechiae during the orthostatic tolerance tests after both bed rest interventions. Importantly, in neither of the campaigns did petechiasis occur during the test before the bed rest intervention or at any other stage of the study. In pre-bed-rest orthostatic tolerance testing of both campaigns, the subject was tilted back at $-40 \mathrm{mmHg}$ due to signs of presyncope.
In post-bed-rest testing, the subject was tilted back at $-10 \mathrm{mmHg}$ in campaign 1 and at $-20 \mathrm{mmHg}$ in campaign 2 . This means that gravitational and LBNP exposure was actually lower during post-bed-rest testing when the petechiae occurred than during pretesting.

Petechial hemorrhages after post-bed-rest testing in both campaigns were distributed throughout the lower legs and most pronounced at the shin in a stocking-like fashion. Petechiae were slightly more pronounced in the first campaign than in the second, though the orthostatic test was stopped at a lower LBNP level in the second campaign $(-10 \mathrm{mmHg}$ and $-20 \mathrm{mmHg})$. Surprisingly, petechiae appeared with a reproducible distribution pattern across campaigns (Figs. 1 and 2). The affected spots remained the same, but varied in intensity. The occurrence of petechiae took place slowly over a time span of several minutes with slow progression during hyperemia.

The evolution after bed rest was without complications. After standing up, the subject walked immediately without problems, and did not report any pain. Petechiae did not cause any pain or discomfort and persisted for about a week before they slowly disappeared.

\section{DISCUSSION}

The mechanism of the adaptation process to $G$ forces resulting in de- or increased petechiae formation is still not fully understood (4). It is furthermore not known what causes the interindividual variance concerning the $G$ threshold of petechiae appearance $(1,7)$. In the presented case, prolonged bed rest decreased the threshold for the occurrence of petechiae and their appearance could be observed post-bed-rest in 1 out of 10 subjects. The subject was a rather active type and $11 \mathrm{yr}$ above the average age of the other subjects in the presented study. A reproducible distribution pattern of petechiae reoccurring in the same locations as shown in this case presentation might indicate that factors predisposing to

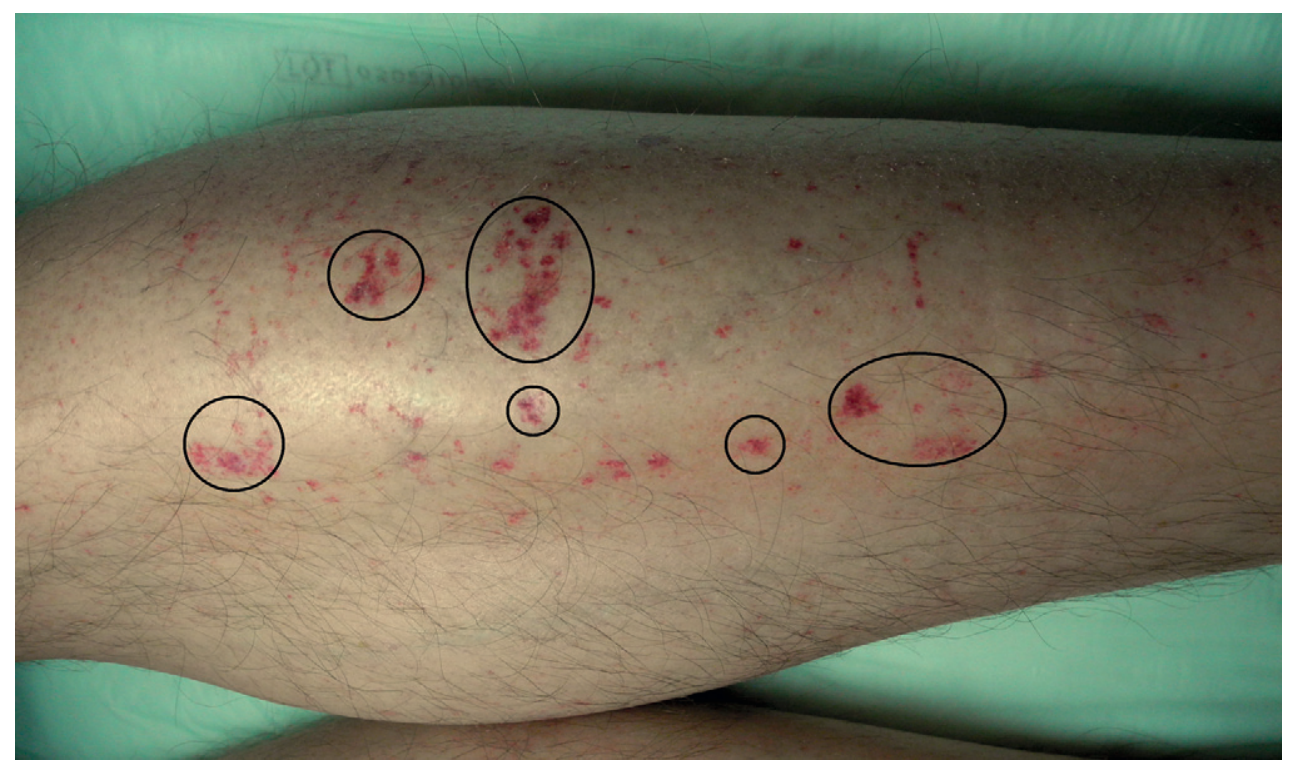

Fig. 1. Petechiae on the shin of the left leg in campaign 1. Circles outline the main spots of petechiae formation. 


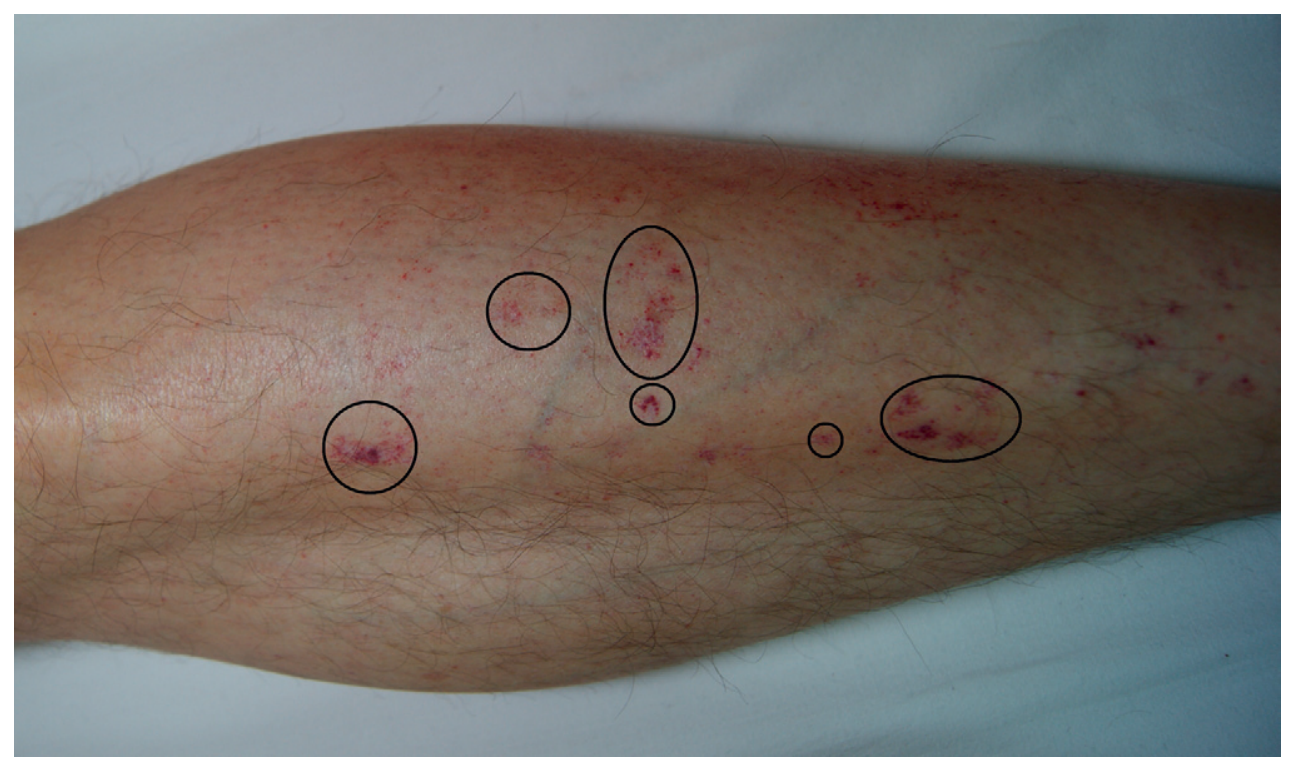

Fig. 2. Petechiae on the shin of the left leg in campaign 2. Hemorrhages are less pronounced than in the first campaign though LBNP pressure was higher. The pattern of distribution shows the same spots as in campaign 1.

petechiae formation keep their local distribution over time. Possibly local vessel structures lead to higher pressure in areas of increased petechiae formation. Effects of hypergravity on hemostasis are known, but it is unclear in how far they influence petechiae formation (3).

Further research regarding effects of microgravity on vessel structures as well as coagulation and hemostasis are indicated to unravel mechanisms leading to petechiae formation. These findings are of clinical relevance as resources can be saved in a clinical setting when a patient develops petechiae in the legs after prolonged bedrest. If petechiae are just located in the lower legs, expensive testing of coagulation and liver parameters as well as thrombocyte functions is not necessarily indicated.

\section{ACKNOWLEDGMENTS}

This study was funded by the European Space Agency, Contract No. 4000105103. The authors would like to thank Dr. Oliver Angerer of ESA, as well as Peter Gauger and Alexandra Noppe (both DLR) for their support.

Authors and affiliations: Bergita Ganse, Dr. med., M.D., Jörn Rittweger, Prof., Dr. med., and Judith Bühlmeier, Ph.D., Dipl. Oec. Troph., Division of Space Physiology, Institute of Aerospace Medicine, German Aerospace Center, Cologne, Germany; Jörn Rittweger, Prof., Dr. med., Institute of Biomedical Research into Human Movement and Health, Manchester Metropolitan University, Manchester, UK; Ulrich Limper, M.D., Department of Anesthesiology and Operative Intensive Care
Medicine, Medical Center Cologne-Merheim, University of Witten/ Herdecke, Cologne, Germany; and Judith Bühlmeier, Ph.D., Dipl. Oec. Troph., Department of Nutrition and Food Science, University of Bonn, Bonn, Germany.

\section{REFERENCES}

1. Abramson DI. Vascular disorders of the extremities, $2^{\text {nd }}$ ed. Hagerstown, MD: Harper and Row; 1974:149.

2. Baisch F, Beck L, Blomqvist G, Wolfram G, Drescher J, Drummer C. Cardiovascular response to lower body negative pressure (LBNP) simulation before, during, and after spaceflight. Eur J Clin Invest 2000; 30:1055-65.

3. Gerzer R. Hypergravity and microgravity influence haemostasis. Thromb Haemost 2009; 101:799.

4. Gustafsson T, Kölegård R, Sundblad P, Norman B, Eiken O. Elevations of local intravascular pressures release vasoactive substances in humans. Clin Physiol Funct Imaging 2013; 33:38-44.

5. Kotovskaia AR, Vil'Vil'iams IF, Elizarov SI, Yliatovskii NV, Gavrilova LN, Liamin VR. [Tolerance of $+\mathrm{Gz}$ loads by space physician Poliakov VV during the active phases of his 438-days space mission.] Aviakosm Ekolog Med 1997; 31:29-34.

6. Kotovskaia AR, Vil'Vil'iams IF, Fomina GA. [Correlation of the cosmonauts' physiological reactions to $+\mathrm{Gx}$ loads during deorbit with the hemodynamic shifts in the period of short-term microgravity.] Aviakosm Ekolog Med 2005; 39:9-15.

7. Whinnery JE. Comparative distribution of petechial haemorrhages as a function of aircraft cockpit geometry. J Biomed Eng 1987; 9:201-5.

8. Wu B, Xue Y, Wu P, Gu Z, Wang Y, Jing X. Physiological responses of astronaut candidates to simulated $+\mathrm{Gx}$ orbital emergency re-entry. Aviat Space Environ Med 2012; 83:758-63. 